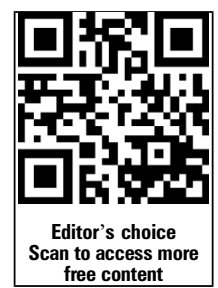

\title{
Efficacy and safety of subcutaneous tabalumab in patients with systemic lupus erythematosus: results from ILLUMINATE-1, a 52-week, phase III, multicentre, randomised, double-blind, placebo-controlled study
}

\author{
D A Isenberg, ${ }^{1}$ M Petri, ${ }^{2}$ K Kalunian, ${ }^{3}$ Y Tanaka, ${ }^{4}$ M B Urowitz, ${ }^{5}$ R W Hoffman, ${ }^{6}$

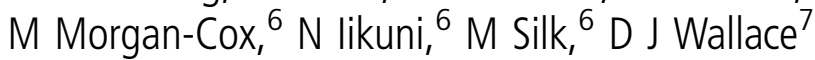

Handling editor Tore K Kvien

- Additional material is published online only. To view please visit the journal online (http://dx.doi.org/10.1136/ annrheumdis-2015-207653).

For numbered affiliations see end of article.

\section{Correspondence to} Professor David A Isenberg, Room 424 The Rayne Building, University College London, 5 University Street, London WC1E 6JF, UK;

d.isenberg@ucl.ac.uk

Received 25 March 2015 Revised 12 August 2015 Accepted 13 August 2015 Published Online First 3 September 2015

- http://dx.doi.org/10.1136/ annrheumdis-2015-207654 - http://dx.doi.org/10.1136/ annrheumdis-2015-208312

To cite: Isenberg DA, Petri M, Kalunian $\mathrm{K}$, et al. Ann Rheum Dis

2016;75:323-331.

\section{ABSTRACT}

Objectives Evaluate efficacy and safety of tabalumab, a human IgG4 monoclonal antibody that binds and neutralises membrane and soluble B-cell activating factor (BAFF) versus placebo plus standard of care (SoC) in patients with systemic lupus erythematosus (SLE). Methods This phase III, 52-week study randomised 1164 patients with moderate-to-severe SLE (Safety of Estrogens in Lupus Erythematosus National Assessment - SLE Disease Activity Index $\geq 6$ at baseline). Patients received SoC plus subcutaneous injections of tabalumab or placebo, starting with a loading dose $(240 \mathrm{mg})$ at week 0 and followed by $120 \mathrm{mg}$ every two weeks (120 Q2W, $n=387), 120 \mathrm{mg}$ every four weeks (120 Q4W, $\mathrm{n}=389$ ) or placebo Q2W ( $\mathrm{n}=388)$. Primary endpoint: proportion of patients achieving SLE Responder Index 5 (SRI-5) response at week 52.

Results Similar proportions of patients in each group achieved SRI-5 response at week 52 (120 Q2W: 31.8\%; 120 Q4W: $35.2 \%$ and placebo: $29.3 \%)$. Key secondary endpoints were not met. In a sensitivity analysis not excluding patients who decreased antimalarials or immunosuppressants, SRI-5 response was achieved with 120 Q4W (37.0\% vs 29.8\% placebo; $p=0.021)$, but not 120 Q2W (34.1\%; $p=0.171)$. Significant reductions in anti-dsDNA antibodies, increases in C3 and C4, and reductions in total $B$ cells and immunoglobulins were observed with tabalumab. No differences were observed between treatment groups in percentage of deaths (120 Q2W: 0.8\%; 120 Q4W: 0.5\%; placebo: 0.5\%), serious adverse events (AEs) (range 11.1-14.4\%) or treatmentemergent AEs (range 81.1-82.3\%).

Conclusions Tabalumab had biological activitychanges in anti-dsDNA, complement, B cells and immunoglobulins - consistent with BAFF pathway inhibition. Key clinical efficacy endpoints did not achieve statistical significance. Safety profiles were similar with tabalumab and placebo.

Trial registration number NCT01196091.

\section{INTRODUCTION}

Systemic lupus erythematosus (SLE) is an autoimmune disease with an estimated prevalence of 7 160 cases in 100000 worldwide. ${ }^{12}$ Mortality has improved, probably due to faster and more accurate diagnosis, increased disease awareness, more appropriate use of corticosteroids and cytotoxic/ immunosuppressive drugs, and a greater focus on treatment of comorbidities. However, few drugs are approved for the treatment of SLE, and a substantial unmet medical need remains.

There is compelling evidence that B cells are involved in the etiopathogenesis of SLE. B cells may be blocked directly by antibodies to CD20 or CD22, or via blockade of vital B-cell survival factors (B-cell activating factor (BAFF) and a proliferation-inducing ligand (APRIL)). ${ }^{3-5}$ BAFF is a tumour necrosis factor family ligand that is important for B-cell development, survival and differentiation. ${ }^{6-8}$ BAFF serum is elevated in patients with SLE and correlate with autoantibody production and disease activity. ${ }^{9} 10$ Thus, targeting B cells by neutralising BAFF may suppress SLE disease activity. Tabalumab is a fully human IgG4 monoclonal antibody, administered subcutaneously, that binds and neutralises both membrane and soluble BAFF. Two phase III studies, ILLUMINATE-1 and ILLUMINATE-2, evaluated the efficacy and safety of subcutaneous doses of tabalumab plus standard of care (SoC) versus placebo plus SoC in patients with active SLE. We present ILLUMINATE-1 results.

\section{METHODS}

\section{Study design}

Patients in ILLUMINATE-1, a 52-week, phase III, multicentre, randomised, double-blind, placebocontrolled study, were randomly assigned 1:1:1 (stratified by anti-dsDNA positivity and African descent) to receive subcutaneous tabalumab $120 \mathrm{mg}$ every two weeks (Q2W) plus SoC (120 Q2W), tabalumab $120 \mathrm{mg}$ every four weeks (Q4W) plus SoC (120 Q4W; administered by alternating $120 \mathrm{mg}$ tabalumab and placebo injections Q2W), or placebo Q2W plus SoC. At week 0, the tabalumab groups received a $240 \mathrm{mg}$ loading dose $(2 \times 120 \mathrm{mg}$ injection) and the placebo group received two placebo injections. Blinded treatments were administered from week 0 through 50. After week 52, patients discontinued study drug and entered safety follow-up or entered a multiyear, open-label, safety extension study of tabalumab. Data from the double-blind, 52-week period are included in this report. 
Patients were allowed to continue SoC for SLE, including non-steroidal anti-inflammatory drugs (unrestricted), corticosteroids, stable doses of antimalarials (such as chloroquine or hydroxychloroquine) and/or stable doses of immunosuppressants (such as azathioprine, methotrexate, mycophenolate, cyclophosphamide or cyclosporine). Increases or decreases in corticosteroid dose were allowed during the first 24 weeks, after which the dose had to be less than or equal to the baseline dose, and only reductions were allowed thereafter. No added, increases in or decreases in antimalarials or immunosuppressants were allowed at any time.

\section{Entry criteria}

Eligible patients were 18 years or older and had a diagnosis of SLE (fulfilled 4/11 American College of Rheumatology revised criteria), ${ }^{11}$ a positive antinuclear antibody (HEp-2 ANA; titre $\geq 1: 80$ ) test by the central laboratory during screening (patients with negative ANA, but positive anti-dsDNA, were retested before being excluded), and a Safety of Estrogens in Lupus Erythematosus National Assessment-SLE Disease Activity Index (SELENA-SLEDAI) score $\geq 6^{12}$ (see online supplementary text).

\section{Endpoints}

The primary endpoint was the proportion of patients achieving an SLE Responder Index 5 (SRI-5) response at week 52. The SRI-5 is a composite endpoint defined as $\geq 5$ point improvement (reduction) in SELENA-SLEDAI score, no new British Isles Lupus Assessment Group (BILAG) 2004 index score of A or no more than one new BILAG B score, and no worsening (increase $\geq 0.3$ points from baseline) in physician's global assessment (PGA). ${ }^{13}$ Patients who did not meet all three clinical criteria, who added, increased or decreased antimalarial or immunosuppressant treatment, who could not adhere to corticosteroiddosing requirements and/or who discontinued study prior to week 52 were considered non-responders.

Key secondary endpoints included time to first severe SLE flare on the SELENA-SLEDAI Flare Index, ${ }^{14}$ proportion of patients with reduction in corticosteroid dose by $\geq 25 \%$ to $\leq 7.5 \mathrm{mg} /$ day prednisone (or equivalent) for $\geq 3$ consecutive months from weeks 24 through 52, and change from baseline on the Brief Fatigue Inventory at week 52. ${ }^{15} 16$
Other endpoints included change in proportion of SRI-5 responders over time; individual components of SRI-5; the proportion of patients achieving SRI-4 and SRI-6 through SRI-10 at week 52; and mean changes in SELENA-SLEDAI, PGA, Systemic Lupus Erythematosus Disease Activity Index 2000, Systemic Lupus International Collaborating Clinics (SLICC) damage index and disease-related biomarkers (anti-dsDNA, C3 and C4) at week 52. A medical team reviewed efficacy endpoint data for consistency. Serum tabalumab concentrations, changes in pharmacodynamic markers (total B cells, B-cell subsets, mean BAFF levels and immunoglobulin concentrations), on the Columbia-Suicide Severity Rating Scale and on the Quick Inventory of Depressive Symptomatology (QIDS-SR16), and other safety assessments (serious adverse events (SAEs), treatment-emergent AEs (TEAEs), AEs of special interest, vital signs, clinical laboratory data) were analysed (see online supplementary text).

\section{Statistical analysis}

All analyses of efficacy, serum tabalumab concentrations and biological activity were conducted on the intent-to-treat population. Safety analyses were applied to the safety population (patients who received $\geq 1$ dose of study drug). For the primary endpoint (SRI-5), a sample size of 380 patients per group was predicted to provide $>95 \%$ power to detect a statistically significant difference of $\geq 14 \%$ between the tabalumab and placebo groups. Statistical methods used a two-sided, 0.05 significance level and included logistic regression for binary efficacy variables, Cox proportional hazard model for time-to-event variables, Fisher's exact for discrete variables and mixed-effects model for repeated measures or analysis of covariance for continuous variables. A graphical approach for multiple comparisons was used to control the overall type I error in analysis of primary and key secondary endpoints. Additional analyses were performed to identify baseline characteristics associated with responders at week 52 and to evaluate how response to tabalumab relative to placebo varied across different categories within a subgroup. Analyses of efficacy variables were adjusted for randomisation stratification factors (anti-dsDNA status and race) and region. For all non-SRI continuous measures, a last observation carried forward (LOCF) approach was used.
Figure 1 Flow diagram of patient disposition during the study. ${ }^{\text {aPatients }}$ from two study sites were not included in the intent-to-treat (ITT) population due to violations at the study site. $A E_{\text {, }}$ adverse event; Q2W, every two weeks; Q4W, every four weeks.

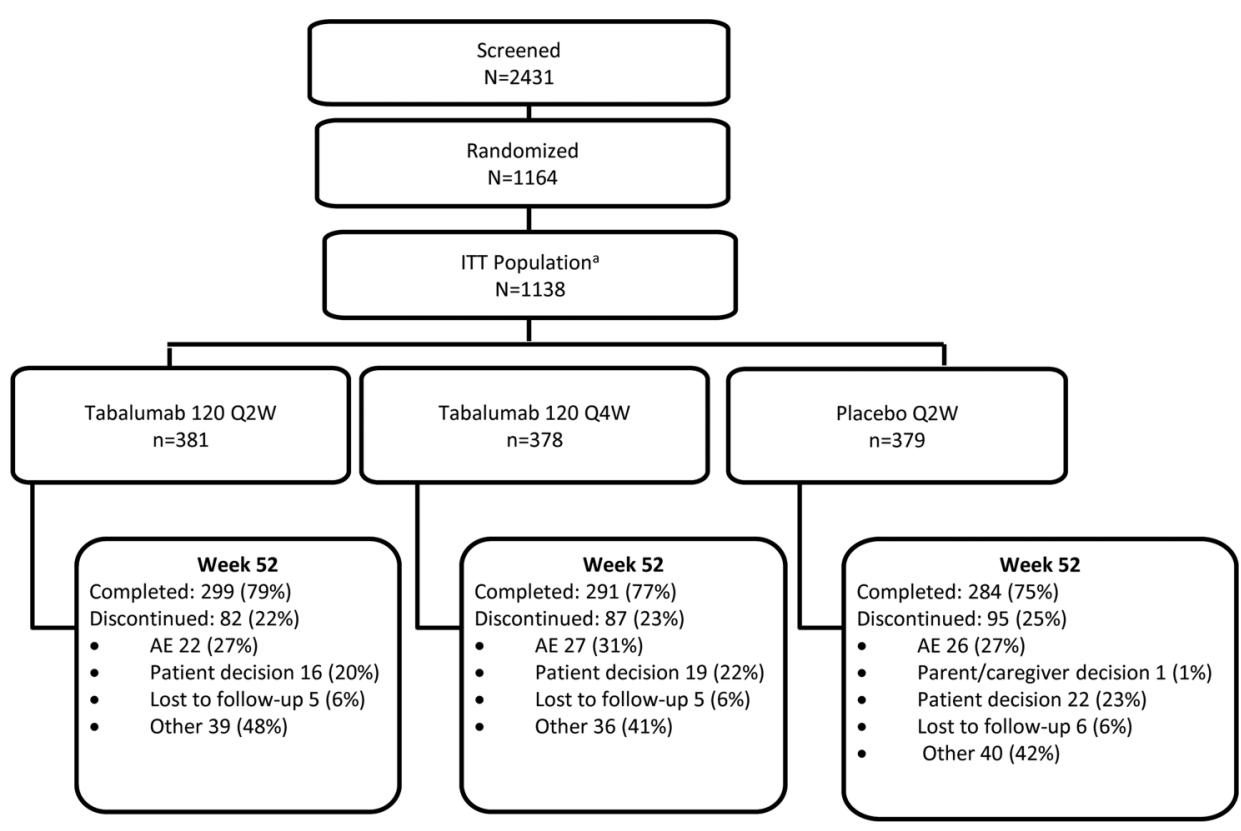

Isenberg DA, et al. Ann Rheum Dis 2016;75:323-331. doi:10.1136/annrheumdis-2015-207653 
Table 1 Baseline demographics and clinical characteristics

\begin{tabular}{|c|c|c|c|}
\hline & $\begin{array}{l}120 \text { Q2W } \\
n=381\end{array}$ & $\begin{array}{l}120 \text { Q4W } \\
n=378\end{array}$ & $\begin{array}{l}\text { Placebo } \\
n=379\end{array}$ \\
\hline Age, years & $40 \pm 13$ & $40 \pm 11$ & $39 \pm 12$ \\
\hline Age, range & $18-87$ & 19-79 & $18-72$ \\
\hline Women, $\mathrm{n}(\%)$ & $354(92.9)$ & $352(93.1)$ & $360(95.0)$ \\
\hline \multicolumn{4}{|l|}{ Race, $\mathrm{n}(\%)$} \\
\hline Indigenous Americant & $65(17.1)$ & $55(14.6)$ & $67(17.7)$ \\
\hline Asian & $68(17.8)$ & $61(16.1)$ & $66(17.4)$ \\
\hline Black/African-American & $40(10.5)$ & $41(10.8)$ & 39 (10.3) \\
\hline Native Hawaiian or Pacific Islander & 0 & $1(0.3)$ & 0 \\
\hline White & $204(53.5)$ & $218(57.7)$ & $205(54.1)$ \\
\hline Multiple races & $4(1.0)$ & $2(0.5)$ & $2(0.5)$ \\
\hline Hispanic/Latino origin, $\mathrm{n}(\%) \ddagger$ & $119(31.2)$ & $116(30.7)$ & $122(32.2)$ \\
\hline \multicolumn{4}{|l|}{ Geographic region, n (\%) } \\
\hline USA/Canada & $133(34.9)$ & $140(37.0)$ & $129(34.0)$ \\
\hline Mexico/Central America/South America & $89(23.4)$ & $84(22.2)$ & $92(24.3)$ \\
\hline Asia Pacific & $66(17.3)$ & $58(15.3)$ & $62(16.4)$ \\
\hline Europe & 74 (19.4) & $77(20.4)$ & 75 (19.8) \\
\hline Africa/Middle East & $19(5.0)$ & $19(5.0)$ & $21(5.5)$ \\
\hline \multicolumn{4}{|l|}{ SLE disease activity } \\
\hline Time since SLE onset, years & $8 \pm 7^{*}$ & $8 \pm 8^{*}$ & $6 \pm 7$ \\
\hline \multicolumn{4}{|l|}{ SELENA-SLEDAI organ system involvement, $\mathrm{n}(\%)$} \\
\hline CNS & $6(1.6)$ & $3(0.8)$ & $6(1.6)$ \\
\hline Vascular & $28(7.3)$ & $29(7.7)$ & $30(7.9)$ \\
\hline Musculoskeletal & $318(83.5)$ & $298(78.8)$ & $308(81.3)$ \\
\hline Renal & $36(9.4)$ & $40(10.6)$ & $42(11.1)$ \\
\hline Mucocutaneous & $344(90.3)$ & $348(92.1)$ & $349(92.1)$ \\
\hline Cardiovascular and respiratory & $27(7.1)$ & $25(6.6)$ & $26(6.9)$ \\
\hline Immunologic & $272(71.4)$ & $284(75.1)$ & $273(72.0)$ \\
\hline Constitutional & $5(1.3)$ & $4(1.1)$ & $5(1.3)$ \\
\hline Haematologic & $39(10.2)$ & $40(10.6)$ & $46(12.1)$ \\
\hline SELENA-SLEDAI score & $10.2 \pm 3.5$ & $10.4 \pm 3.6$ & $10.7 \pm 3.9$ \\
\hline SELENA-SLEDAI $\geq 10, \mathrm{n}(\%)$ & $222(58.3)$ & $219(57.9)$ & $224(59.1)$ \\
\hline SLEDAI-2K score & $10.6 \pm 3.7$ & $10.7 \pm 3.9$ & $10.8 \pm 4.0$ \\
\hline$\geq 1 \mathrm{~A}$ or $2 \mathrm{~B}$ BILAG scores, $n(\%)$ & $228(59.8)$ & $228(60.3)$ & $220(58.2)$ \\
\hline PGA score & $46.3 \pm 15.7$ & $46.1 \pm 16.2$ & $47.1 \pm 16.1$ \\
\hline SLICC damage index & $0.5 \pm 1.0^{*}$ & $0.6 \pm 1.2^{*}$ & $0.4 \pm 0.8$ \\
\hline \multicolumn{4}{|l|}{ Medications } \\
\hline Prednisone, $\mathrm{n}(\%)$ & $300(78.7)$ & $296(78.3)$ & $295(77.8)$ \\
\hline Mean prednisone dose (or equivalent), mg/day & $11.9 \pm 7.4$ & $12.1 \pm 7.8$ & $12.1 \pm 7.9$ \\
\hline Prednisone $>7.5 \mathrm{mg} /$ day, $\mathrm{n}(\%)$ & $197(51.7)$ & $200(52.9)$ & $196(51.7)$ \\
\hline Antimalarial, $\mathrm{n}(\%)$ & $241(63.3)$ & $241(63.8)$ & $243(64.1)$ \\
\hline Any immunosuppressant, $\mathrm{n}(\%)$ & $158(41.5)$ & $176(46.6)$ & $164(43.3)$ \\
\hline MMF, n (\%) & $24(6.3)$ & $38(10.1)$ & $32(8.4)$ \\
\hline AZA, n (\%) & $66(17.3)$ & $80(21.2)$ & $80(21.1)$ \\
\hline MTX, n (\%) & $50(13.1)$ & $40(10.6)$ & 40 (10.6) \\
\hline \multicolumn{4}{|l|}{ Biomarkers and other laboratory measures } \\
\hline$\leq 45 \mathrm{mg} / \mathrm{mmol}$ & $330(86.8)$ & $326(86.2)$ & $332(87.6)$ \\
\hline$>45 \mathrm{mg} / \mathrm{mmol}$ & $50(13.2)$ & $52(13.8)$ & $47(12.4)$ \\
\hline Total B-cell counts, median (range), cells $/ \mu \mathrm{L}$ & $131.5(3-1979)$ & $128.0(6-1727)$ & $129.5(3-1345)$ \\
\hline BAFF concentration, $\mathrm{pg} / \mathrm{mL}$ & $2559 \pm 8277$ & $2230 \pm 4230$ & $1753 \pm 2439$ \\
\hline Anti-dsDNA $\geq 30 \mathrm{IU} / \mathrm{mL}, \mathrm{n}(\%) \S$ & $228(59.8)$ & $231(61.1)$ & $226(59.6)$ \\
\hline Anti-dsDNA, IU & $107.2 \pm 113.5$ & $110.4 \pm 111.6$ & $107.1 \pm 112.4$ \\
\hline C3 $<$ LLN, n (\%) & $144(37.9)$ & $150(39.8)$ & $163(43.1)$ \\
\hline $\mathrm{C} 3, \mathrm{~g} / \mathrm{L}$ & $1.0 \pm 0.3$ & $1.0 \pm 0.3$ & $1.0 \pm 0.3$ \\
\hline
\end{tabular}




\begin{tabular}{|c|c|c|c|}
\hline & $\begin{array}{l}120 \text { Q2W } \\
n=381\end{array}$ & $\begin{array}{l}120 Q 4 W \\
n=378\end{array}$ & $\begin{array}{l}\text { Placebo } \\
n=379\end{array}$ \\
\hline C4 <LLN, n (\%) & $84(22.1)$ & $85(22.5)$ & $98(25.9)$ \\
\hline $\mathrm{C} 4, \mathrm{~g} / \mathrm{L}$ & $0.2 \pm 0.1$ & $0.2 \pm 0.1$ & $0.2 \pm 0.1$ \\
\hline $\operatorname{lgG}, g / L$ & $15.6 \pm 5.7$ & $15.7 \pm 5.9$ & $15.6 \pm 5.6$ \\
\hline $\lg \mathrm{G}<\mathrm{LLN}, \mathrm{n}(\%)$ & $2(0.5)$ & $1(0.3)$ & $2(0.5)$ \\
\hline $\lg M, g / L$ & $1.1 \pm 0.7$ & $1.2 \pm 0.8$ & $1.2 \pm 1.0$ \\
\hline $\operatorname{lgM}<\mathrm{LLN}, \mathrm{n}(\%)$ & $36(9.4)$ & $34(9.0)$ & $35(9.2)$ \\
\hline $\lg A, g / L$ & $3.1 \pm 1.6$ & $3.2 \pm 1.6$ & $3.1 \pm 1.5$ \\
\hline $\lg \mathrm{A}<\mathrm{LLN}, \mathrm{n}(\%)$ & $10(2.6)$ & $7(1.9)$ & $11(2.9)$ \\
\hline \multicolumn{4}{|l|}{ uPCR, n (\%) } \\
\hline \multicolumn{4}{|c|}{$\begin{array}{l}\text { * } \mathrm{p}<0.05, \mathrm{p} \text { value for comparison of treatment groups derived using an analysis of variance model with treatment group and geographic region fitted as explanatory variables. } \\
\text { †American Indian from North, Central and South America and Alaska Native. } \\
\text { fEthnicity (Hispanic/Latino) information was only required to be collected in the USA. } \\
\text { §Or }>10 \text { IU/mL for patients for whom the original DNA autoantibody assay was performed. } \\
\text { Anti-dsDNA, anti-double-stranded DNA; AZA, azathioprine; BAFF, B-cell activating factor; BILAG, British Isles Lupus Assessment Group index; CNS, central nervous system; Ig, } \\
\text { immunoglobulin; LLN, lower limit of normal; MMF, mycophenolate mofetil; MTX, methotrexate; PGA, physician's global assessment; Q2W, every two weeks; Q4W, every four weeks; } \\
\text { SELENA-SLEDAl, Safety of Estrogens in Lupus Erythematosus National Assessment-Systemic Lupus Erythematosus Disease Activity Index; SLE, systemic lupus erythematosus; SLEDAI-2K, } \\
\text { Systemic Lupus Erythematosus Disease Activity Index 2000; SLICC, Systemic Lupus International Collaborating Clinics; uPCR, urine protein to urine creatinine ratio. }\end{array}$} \\
\hline
\end{tabular}

\section{RESULTS}

\section{Patient disposition and baseline characteristics}

Of the 1164 randomised patients, $\geq 75 \%$ in each group completed the 52-week trial (figure 1). Discontinuation rates and reasons for early discontinuation were similar across groups. Baseline demographics, SLE disease activity and medications were generally balanced across groups (table 1).

\section{Efficacy}

The primary endpoint, SRI-5 response at week 52, was not achieved in either treatment group (figure $2 \mathrm{~A}$, table 2). At week
52, SRI-5 (non-responder imputation (NRI)) response rates were $31.8 \%$ in the $120 \mathrm{Q} 2 \mathrm{~W}$ and $35.2 \%$ in the $120 \mathrm{Q} 4 \mathrm{~W}$ group versus $29.3 \%$ in the placebo group $(\mathrm{p}>0.05$, each comparison). For all three components of SRI, the percentages of responders were higher in the tabalumab groups versus placebo (table 2). See online supplementary table S1 for specific SELENA-SLEDAI organ improvement.

None of the key secondary endpoints (ie, time to first severe SLE flare, corticosteroid-sparing effects and change from baseline in worst level of fatigue) were significantly different with tabalumab versus placebo (table 2). At week 52, the changes in
A

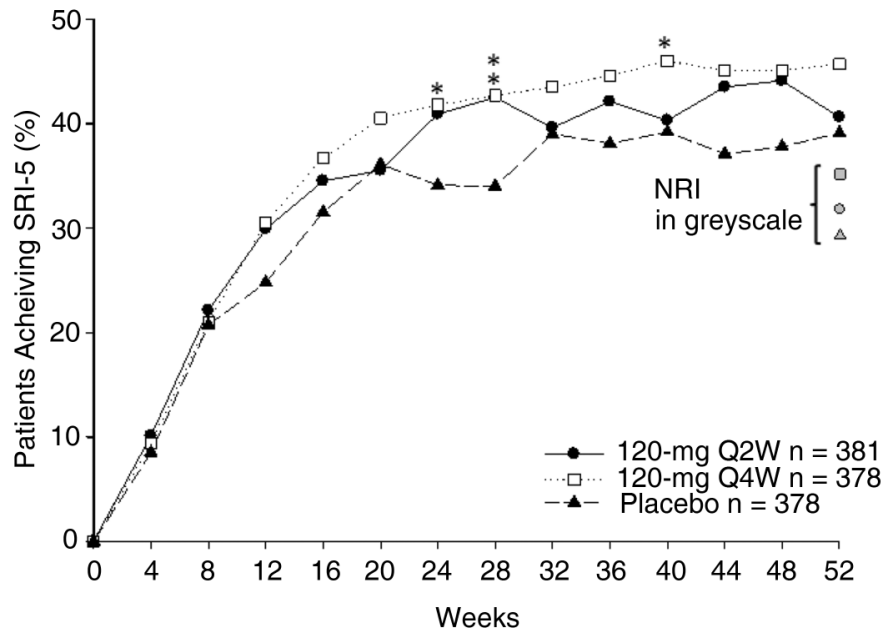

B

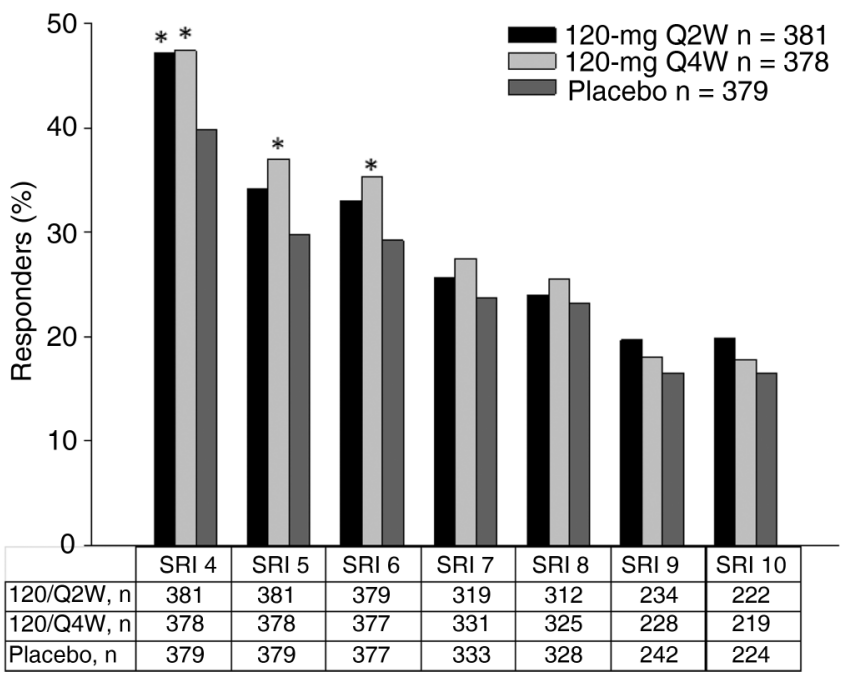

Figure 2 Clinical outcomes. (A) SLE Responder Index 5 (SRI-5) response rates over the 52-week treatment period. Primary endpoint analysis included patients who achieved SRI-5 endpoint without violating the concomitant medication rules. Patients who did not meet all three clinical criteria, who added, increased or decreased antimalarial or immunosuppressant treatment, who could not meet the corticosteroid-dosing requirements and/or who discontinued the study prior to week 52 were considered non-responders. $p$ Value based on odds ratio estimated from logistic regression model. (B) SRI-4 through SRI-10 response rates at week 52. Predefined analyses of SRI endpoints described above used a modified concomitant medication rule where patients who decreased their antimalarials or immunosuppressants during the study were not automatic non-responders. SRI-6 through SRI-10 performed in subset of patients who entered study with Safety of Estrogens in Lupus Erythematosus National Assessment-SLE Disease Activity Index (SELENA-SLEDAI) $\geq 6$-SELENA-SLEDAI $\geq 10$, respectively. ${ }^{*} p \leq 0.05$ vs placebo; ${ }^{*} p \leq 0.001$ vs placebo. Symbols in greyscale represent 52 -week endpoint data calculated using a non-responder imputation (NRI), circle=120 every two weeks $(\mathrm{Q} 2 \mathrm{~W})$, square=120 every four weeks $(\mathrm{Q} 4 \mathrm{~W})$ and triangle $=$ placebo. 
SELENA-SLEDAI scores were also similar in the 120 Q2W (least-squares (LS) mean: -5.1), $120 \mathrm{Q} 4 \mathrm{~W}$ (LS mean: -5.4) and placebo groups (LS mean: -5.2 ).

In a predefined sensitivity analysis of the primary endpoint, where patients who decreased their antimalarials or immunosuppressants during the study were not automatic non-responders ${ }^{17}$ SRI-5 (NRI) response rates were $34.1 \%$ in the 120 Q2W $(\mathrm{p}=0.171)$ and $37.0 \%$ in the $120 \mathrm{Q} 4 \mathrm{~W}$ group $(\mathrm{p}=0.021)$ versus $29.8 \%$ in the placebo group at week 52 (figure $2 \mathrm{~B}$ ). In the same predefined analyses of SRI-4 and SRI-6 through SRI-10, both the 120 Q2W (47.2\%) and 120 Q4W groups $(47.4 \%)$ had a significantly higher percentage of SRI-4 responders at week 52 versus placebo $(39.8 \% ; \mathrm{p} \leq 0.05$, both comparisons), and significantly more 120 Q4W patients achieved SRI-6 response versus placebo $(35.3 \%$ vs $29.2 \%, \mathrm{p}=0.044)$ at week 52.

The effect of tabalumab was also evaluated in patients who were anti-dsDNA positive and who had low C3 and C4 levels at baseline. There was no significant difference between tabalumab and placebo in percentage of SRI-5 responders at week $52(120$ Q2W: 34.1\%, 120 Q4W: 32.3\%, placebo: 35.4\%; p >0.05, each comparison).

The anti-dsDNA levels decreased significantly in both tabalumab groups versus placebo as early as week 4 (figure 3) and continued to decrease and remained well below baseline levels through week 52 (LS mean changes, 120 Q2W: -25.60 IU; 120 Q4W: -22.28 IU; placebo: -4.13 IU; $p<0.001$, each comparison). Of the patients with anti-dsDNA $\geq 30 \mathrm{IU} / \mathrm{mL}$ (positive) at

Table 2 Clinical and biomarker outcomes

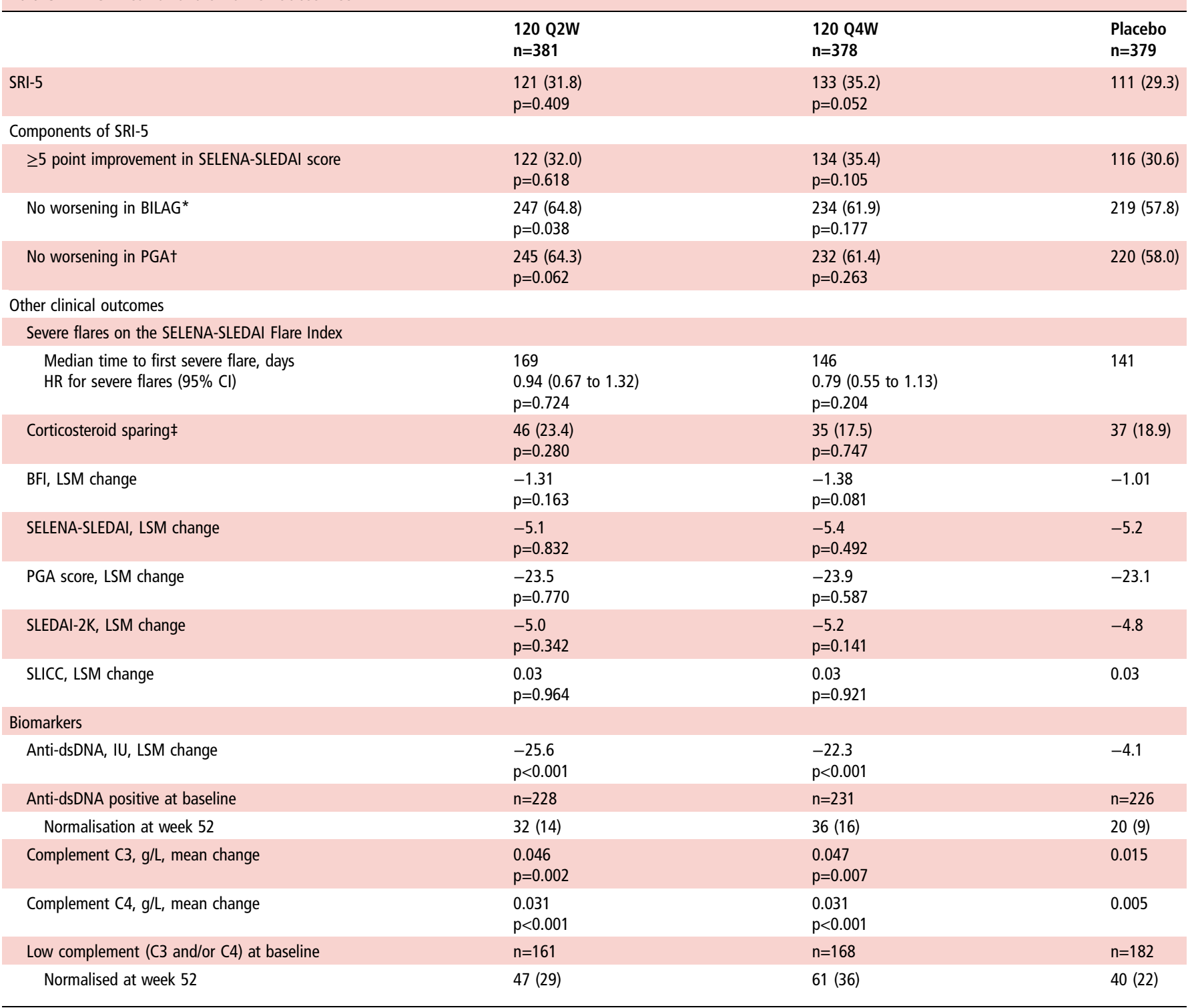

All data are $n(\%)$ unless indicated otherwise.

*No new BILAG A and no more than one new BILAG B.

tWorsening defined as an increase of $\geq 0.3$ points from baseline.

$\ddagger$ Percent of patients able to decrease corticosteroid dose by at least $25 \%$ to $\leq 7.5 \mathrm{mg} /$ day prednisone for at least three consecutive months from weeks 24 to 52 without an increase in dose of either antimalarials or immunosuppressants at any time during the treatment period.

Anti-dsDNA, anti-double-stranded DNA; BFI, Brief Fatigue Inventory; BILAG, British Isles Lupus Assessment Group index; LSM, least-squares mean change from baseline, PGA, physician's global assessment; Q2W, every two weeks; Q4W, every four weeks; SELENA-SLEDAI, Safety of Estrogens in Lupus Erythematosus National Assessment-Systemic Lupus Erythematosus Disease Activity Index; SLE, systemic lupus erythematosus; SLEDAI-2K, Systemic Lupus Erythematosus Disease Activity Index 2000; SLICC, Systemic Lupus International Collaborating Clinics; SRI-5, SLE Responder Index 5. 
Figure 3 Mean change in anti-dsDNA over the 52-week treatment period. * $p \leq 0.05$ vs placebo; ${ }^{* *} p \leq 0.001$ vs placebo. LS, least squares; Q2W, every two weeks; Q4W, every four weeks.

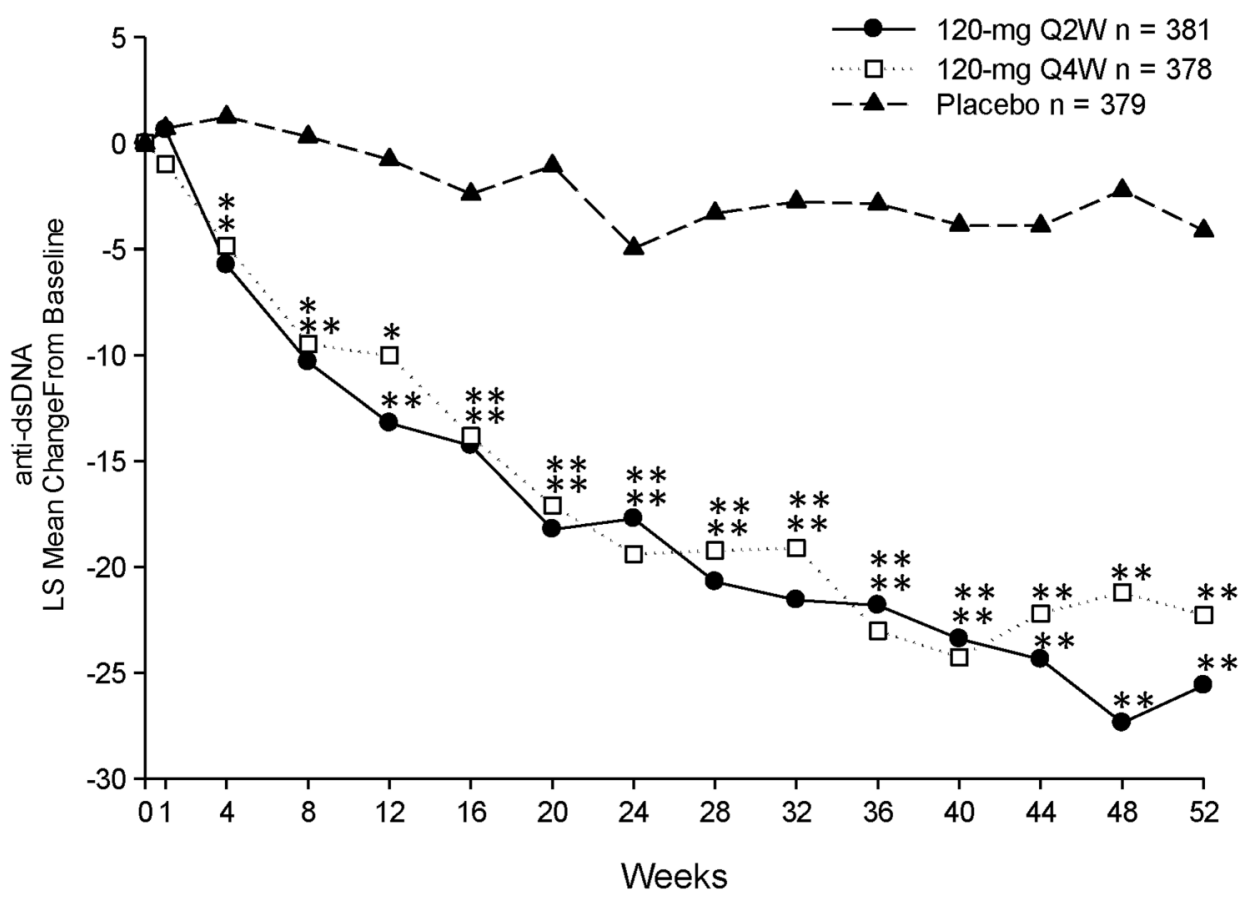

IgA: $2(0.5 \%), 4(1.1 \%)$ and $2(0.5 \%)$; IgG: $4(1.1 \%), 2(0.5 \%)$ and $2(0.5 \%)$; and IgM: $64(18.6 \%), 52(15.2 \%)$ and 14 $(4.0 \%)$, respectively.

placebo returned to normal levels $(<30 \mathrm{IU} / \mathrm{mL})$ at week 52 .

Increases in C3 and C4 were observed in both tabalumab groups versus placebo at week 52 (table 2). Of the patients with low complement C3 and/or C4 at baseline, 29\% in $120 \mathrm{Q} 2 \mathrm{~W}$ and $36 \%$ in the $120 \mathrm{Q} 4 \mathrm{~W}$ group versus $22 \%$ in the placebo group had normalised at week 52 .

Other than sex, there were no baseline variables (ie, age, region, anti-dsDNA status, complement, race, ethnicity, disease severity (SELENA-SLEDAI $<10$ or $\geq 10$ ), mucocutaneous or musculoskeletal organ involvement, PGA score, SLICC score, severe seropositivity, concomitant medication use, number of prior treatment failures, comorbidities, time since onset of SLE or BAFF concentration) for which the treatment effect differed across subgroups.

\section{Pharmacodynamics}

At week 52, tabalumab treatment was associated with increases in median BAFF concentrations from baseline (120 Q2W: $95349 \mathrm{pg} / \mathrm{mL}$; $120 \mathrm{Q} 4 \mathrm{~W}: 91332 \mathrm{pg} / \mathrm{mL}$ ); as expected, these increases were significantly different from changes observed in the placebo group ( $27 \mathrm{pg} / \mathrm{mL}, \mathrm{p}<0.001$, both comparisons) due to presence of active drug binding to BAFF. In the tabalumab groups, median total B-cell counts initially increased from baseline at week 1 (120 Q2W: 64.5 cells/ $\mu \mathrm{L} ; 120 \mathrm{Q} 4 \mathrm{~W}: 70.0$ cells/ $\mu \mathrm{L}$; placebo: -1.0 cells $/ \mu \mathrm{L} ; \mathrm{p}<0.001$, each comparison) and subsequently decreased below baseline values through week 52 (LOCF) (120 Q2W: -68.0 cells/ $\mu \mathrm{L} ; 120 \mathrm{Q} 4 \mathrm{~W}:-57.0$ cells/ $\mathrm{LL}$; placebo: -7.5 cells $/ \mu \mathrm{L} ; \mathrm{p}<0.001$, each comparison) (see online supplementary figure S1A). B-cell subset changes in both tabalumab groups were significantly different from placebo; however, there was no clear dose separation in either tabalumab group. The largest magnitude of change for B cells was the reduction in the mature naive subset. No significant T-cell changes were observed. Statistically significant decreases in serum immunoglobulins were observed at each time point over the 52-week treatment period (see online supplementary figure S1 B,D). However, the number of patients who dropped below normal range was low in the $120 \mathrm{Q} 2 \mathrm{~W}, 120 \mathrm{Q} 4 \mathrm{~W}$ and placebo groups;

\section{Pharmacokinetics}

Analysis of the pharmacokinetic data indicated that tabalumab concentrations shifted higher for the tabalumab 120 Q2W group. Pharmacokinetic analysis also showed an overlap between the two dosing regimens, as expected (data not shown).

\section{Safety and tolerability}

No clinically meaningful or significant differences in the number of deaths, frequency of SAEs or TEAEs, or number of patients who discontinued due to an AE were observed between groups (table 3). The majority of TEAEs were mild-to-moderate. Ten pregnancies were reported during the treatment period (table 3), five of which were carried to term and there were no fetal abnormalities at birth (one in $120 \mathrm{Q} 2 \mathrm{~W}$, one in placebo, three in $120 \mathrm{Q} 4 \mathrm{~W}$ ). Two fetal deaths in utero (one placebo patient with preeclampsia and one patient in 120 Q2W group), one spontaneous abortion (placebo) and one terminated pregnancy (placebo) were reported; and one pregnant patient was lost to follow-up (120 Q2W).

The incidences of serious infections and severe infections were similar in the tabalumab and placebo groups (table 3). The incidences of opportunistic infections of interest were herpes zoster, reported in 13 patients (3.4\%) in $120 \mathrm{Q} 2 \mathrm{~W}, 4(1.0 \%)$ in $120 \mathrm{Q} 4 \mathrm{~W}$ and $10(2.6 \%)$ in placebo; disseminated tuberculosis, reported in 1 patient $(0.3 \%)$ in $120 \mathrm{Q} 2 \mathrm{~W}$; and cytomegalovirus, reported in 1 patient $(0.3 \%)$ in 120 Q4W. Oral candidiasis was reported in six $(1.6 \%)$ patients in $120 \mathrm{Q} 2 \mathrm{~W}$, three $(0.8 \%)$ in 120 Q4W and eight $(2.1 \%)$ in placebo; however, no unexpected fungal opportunistic infections were reported. There were no differences in the percentages of patients reporting an injection-site reaction, in the incidences of adjudicated major adverse cardiovascular events or in reported malignancies. No anaphylactic events were reported; 


\begin{tabular}{|c|c|c|c|}
\hline n (\%) & $\begin{array}{l}120 \text { Q2W } \\
n=386\end{array}$ & $\begin{array}{l}120 Q 4 W \\
n=389\end{array}$ & $\begin{array}{l}\text { Placebo } \\
n=387\end{array}$ \\
\hline Deaths & $3(0.8)^{*}$ & $2(0.5) \dagger$ & $2(0.5) \ddagger$ \\
\hline Serious AEs & $43(11.1)$ & $56(14.4)$ & $50(12.9)$ \\
\hline Discontinued due to an $\mathrm{AE}$ & $26(6.7)$ & $29(7.5)$ & $28(7.2)$ \\
\hline Treatment-emergent AEs & $317(82.1)$ & $320(82.3)$ & $314(81.1)$ \\
\hline AEs possibly related to study drug & $175(45.3)$ & $159(40.9)$ & $161(41.6)$ \\
\hline \multicolumn{4}{|l|}{ Selected treatment-emergent AEs } \\
\hline Pregnancies & $3(0.8)$ & $3(0.8)$ & $4(1.0)$ \\
\hline \multicolumn{4}{|l|}{ Infections } \\
\hline Serious & $20(5.2)$ & $19(4.9)$ & $17(4.4)$ \\
\hline Severe & $10(2.6)$ & $14(3.6)$ & $15(3.9)$ \\
\hline Adjudicated MACE & $5(1.3)$ & $4(1.0)$ & $5(1.3)$ \\
\hline Malignancies & $1(0.3) \S$ & $1(0.3) \Re$ & $2(0.5)^{* *}$ \\
\hline Injection-site reactions & $11(2.8)$ & $7(1.8)$ & $6(1.6)$ \\
\hline \multicolumn{4}{|l|}{ Allergic/hypersensitivity reactions } \\
\hline Anaphylaxis & 0 & 0 & 0 \\
\hline Non-anaphylaxis hypersensitivity & $5(1.3)$ & $1(0.3)$ & $2(0.5)$ \\
\hline \multicolumn{4}{|l|}{ Depression and suicide-related events } \\
\hline Depression & $12(3.1)$ & $14(3.6)$ & $13(3.4)$ \\
\hline Suicide attempt & 0 & $1(0.3)$ & $1(0.3)$ \\
\hline \multicolumn{4}{|l|}{ Columbia-Suicide Severity Rating Scalet† } \\
\hline Suicidal ideation & $1(0.4)$ & $3(1.3)$ & $2(0.9)$ \\
\hline Suicidal behaviour & 0 & $1(0.4)$ & $2(0.9)$ \\
\hline Change in QIDS-SR16 total score, mean \pm SD & $-1.2 \pm 4.09$ & $-1.4 \pm 4.37$ & $-1.4 \pm 4.58$ \\
\hline \multicolumn{4}{|l|}{ Immunogenicity } \\
\hline TE persistent or transient ADA & $2(0.5)$ & $3(0.8)$ & $12(3.1)$ \\
\hline Positive neutralising ADA & 0 & 0 & 0 \\
\hline
\end{tabular}

Data are $\mathrm{n}(\%)$ unless indicated otherwise.

*Antiphospholipid syndrome, pulmonary embolism, disseminated tuberculosis.

†Pneumonia, SLE.

$\ddagger$ Preferred term=death (verbatim reported by investigator was unknown cause of death), CVA.

$\S$ Basal cell carcinoma.

ISquamous cell carcinoma of the skin.

**Breast cancer, intestinal adenocarcinoma.

††Number of patients with at least one post-baseline assessment.

ADA, antidrug antibody; AE, adverse event; CVA, cerebrovascular accident; MACE, major adverse cardiovascular event; Q2W, every two weeks; Q4W, every four weeks; QIDS-SR16, Quick Inventory of Depressive Symptomatology (16-Item); SLE, systemic lupus erythematosus; TE, treatment-emergent.

hypersensitivity reactions occurred in five patients in $120 \mathrm{Q} 2 \mathrm{~W}$, one patient in $120 \mathrm{Q} 4 \mathrm{~W}$ and two patients in placebo.

There were no differences in the percentages of patients in the tabalumab groups versus placebo who reported depression, treatment-emergent suicidal ideation or suicidal behaviour during the treatment period (table 3 ). The change from baseline in the QIDS-SR16 score at week 52 (LOCF) was similar in the tabalumab and placebo groups.

No clinically meaningful differences in laboratory abnormalities were noted. The frequency of treatment-emergent antidrug antibodies was low, and no positive neutralising antidrug antibodies were identified (table 3 ).

\section{DISCUSSION}

This phase III, placebo-controlled study in patients with active, moderate-to-severe SLE assessed the efficacy of tabalumab 120 Q2W or 120 Q4W versus placebo. The primary endpoint, SRI-5 response, was not met for either dose group, though 120 Q4W showed a trend towards improvement versus placebo at week $52(\mathrm{p}=0.052)$.

Statistical significance was not achieved on secondary measures of clinical efficacy (ie, time to first severe flare, corticosteroid-sparing effects, fatigue and individual components of the SRI). However, a significant pharmacodynamic effect (ie, anti-dsDNA reductions, C3 and C4 increases) was observed in the tabalumab groups. In a flare prevention trial, patients administered atacicept (a fusion protein that blocks B-lymphocyte stimulator and APRIL) showed marked antidsDNA reductions and C3 increases, although only patients receiving the higher dose demonstrated any clinical benefit. ${ }^{18}$ The reasons for the difference are not obvious. One could speculate that some form of 'threshold effect' is taking place. In general, biomarkers often perform rather variably in their relationship with clinical disease. Based on clinical observations from this trial and ILLUMINATE- $2,{ }^{17}$ a similarly designed phase III, global study of tabalumab where the SRI-5 endpoint was met and a dose-effect was observed, it remains possible that higher doses or more frequent dosing may lead to better efficacy despite maximal effect on pharmacodynamic markers.

Significant increases in BAFF concentrations, reductions in total $\mathrm{B}$ cells and reductions in immunoglobulins were also observed in the tabalumab groups. These changes were consistent with tabalumab's mechanism of action and with previous studies. ${ }^{19-21}$ Increases in BAFF concentration were expected. In the presence of 
tabalumab, BAFF concentration increases because the anti-BAFF Ig molecule (tabalumab) binds to BAFF creating a BAFF-anti-BAFF Ig complex. The measurable level of BAFF accumulated in the presence of tabalumab. Overall, these outcomes were indicative of biological activity consistent with BAFF inhibition.

Baseline BAFF concentrations were not predictive of higher SRI response rates with tabalumab. A study evaluating whether serum BAFF levels correlate with SLE relapse or remission following B-cell-depleting therapy demonstrated that patients were more likely to flare when serum BAFF was rising. ${ }^{22}$ However, serum BAFF level did not predict subsequent remission or relapse after treatment. Despite being a strong correlate of flares, baseline BAFF concentrations may not be a strong indicator of response to B-cell-targeting therapies.

In a predefined sensitivity analysis of the primary endpoint, where patients who decreased antimalarials or immunosuppressants were not automatically non-responders, SRI-5 response was met with 120 Q4W (37.0\% vs $29.8 \%$ placebo), but not the more frequent 120 Q2W dose (34.1\%). In similar analyses of SRI-4 and SRI-6 through SRI-10, both the 120 Q2W (47.2\%) and 120 Q4W groups $(47.4 \%)$ had a significantly higher SRI-4 response than placebo (39.8\%) and the 120 Q4W group also had a higher SRI-6 response $(35.3 \%$ vs $29.2 \%$ placebo). The ILLUMINATE-2 primary endpoint applied the same concomitant medication rules noted above and SRI-5 response was met with the more frequent 120 Q2W dose, but not $120 \mathrm{Q}^{4} \mathrm{~W}^{18}{ }^{18}$ Additionally, $120 \mathrm{Q} 2 \mathrm{~W}$ also had a significantly higher percentage of responders than placebo when using other SRI cut-offs (ie, SRI-4, SRI-6, SRI-7, SRI-9 and SRI-10).

Belimumab is a monoclonal antibody that targets soluble BAFF and has been approved for the treatment of SLE in a number of countries. ${ }^{23} 24$ Tabalumab is a monoclonal antibody that targets both membrane and soluble BAFF. Beyond the differences in mechanism of action, there are no data that further differentiate the biological activity of these drugs. In two phase III trials of belimumab, $43 \%$ and $58 \%$ of patients, respectively, receiving belimumab $10 \mathrm{mg} / \mathrm{kg}$ plus SoC achieved the primary endpoint of SRI-4 (vs 33\% and 44\%, placebo). ${ }^{25}{ }^{26}$ Unlike the ILLUMINATE-1 study, the belimumab trials allowed the addition of new antimalarial treatments and increases in immunosuppressants up to week 16, and corticosteroid use was less restrictive. Despite similarities in trial design, it is difficult to determine if any dissimilarities in baseline variables, background medications or mechanism of action had an impact on the different study outcomes.

The safety data show no unexpected events and low levels of antidrug antibody. Although a higher incidence of herpes zoster in the Q2W group was noted, there were no other indications of opportunistic infection. Overall, there was no increased risk of mortality, or of serious, severe adverse effects with tabalumab relative to placebo.

This study enrolled patients that principally had joint and skin manifestations, which may limit applicability of the results to the broader, more heterogeneous features of SLE. In addition, variations in clinical practices worldwide were indicated by a relatively small percentage of patients $(\sim 63 \%)$ taking an antimalarial at baseline as well as other differences in SoC. Although baseline variables were balanced across groups, it is possible that differences in SoC may also represent differences in degrees of illness or a patient's responsiveness to treatment. Additionally, in the absence of mild-or-moderate flare analyses, the impact of treatment on flares of lesser severity is unknown.
Tabalumab did not meet the primary endpoint, SRI-5 response, or key secondary endpoints, despite pharmacodynamic evidence of biological activity that was consistent with BAFF pathway inhibition. Safety profiles of the tabalumab groups were similar to placebo with no unexpected signals.

\section{Author affiliations}

${ }^{1}$ University College Hospital to University College London, London, UK

${ }^{2}$ Johns Hopkins University School of Medicine, Baltimore, Maryland, USA

${ }^{3}$ Division of Rheumatology, Allergy and Immunology, UCSD School of Medicine, La Jolla, California, USA

${ }^{4}$ The First Department of Internal Medicine, University of Occupational and Environmental Health, Kitakyushu, Fukuoka, Japan

${ }^{5}$ University of Toronto, Toronto Western Hospital, Toronto, Canada

${ }^{6}$ Eli Lilly and Company, Indianapolis, Indiana, USA

${ }^{7}$ Cedars-Sinai Medical Center, David Geffen School of Medicine, University of California, Los Angeles, California, USA

Acknowledgements The authors would like to thank all of the ILLUMINATE-1 investigators for their participation, Dr Pierre-Yves Berclaz of Eli Lilly and Company (Indianapolis, IN) for assistance with trial design and execution, Rebecca Taha, Steven Watts, Tammy Forrester and Allen Nyhuis of Eli Lilly and Company (Indianapolis, IN) for assistance with statistical analyses, and Kelly Guerrettaz and Cindi Wood of inVentiv Health Clinical (Princeton, NJ) for assistance with manuscript writing and editing, respectively.

Contributors DAl, MBU and KK have participated in the analysis and interpretation of data and drafting and/or critical revision of the manuscript. YT has participated in study conception and design, acquisition of data, analysis and interpretation of the data and critical revision of the manuscript. MM-C has participated in study conception and design, statistical analysis and interpretation of data, and critical revision of the manuscript. MP, RWH, NI, MS and DJW have participated in study conception and design, analysis and interpretation of the data, and drafting and/or critical revision of the manuscript.

\section{Funding Eli Lilly and Company.}

Competing interests DAI has acted as a consultant for Eli Lilly and Company. The honoraria offered are passed to a local arthritis charity. MP has acted as a consultant for Eli Lilly and Company. KK has acted as a consultant for Eli Lilly and Company. YT has received consulting fees, speaking fees and/or honoraria from AbbVie, Daiichi-Sankyo, Chugai, Takeda, Mitsubishi-Tanabe, Bristol-Myers, Astellas, Eisai, Janssen, Pfizer, Asahi-kasei, Eli Lilly and Company, GlaxoSmithKline, UCB, Teijin, MSD, Santen and has received research grants from Mitsubishi-Tanabe, Takeda, Chugai, Astellas, Eisai, Taisho-Toyama, Kyowa-Kirin, AbbVie, Bristol-Myers. MBU has acted as a consultant for Eli Lilly and Company. RWH, MM-C, NI and MS are employees of and are shareholders in Eli Lilly and Company. DJW has acted as a consultant for Eli Lilly and Company.

\section{Patient consent Obtained.}

Ethics approval The protocol was approved by each institutional review board subject to applicable laws and regulations and ethical principles consistent with the Declaration of Helsinki.

Provenance and peer review Not commissioned; externally peer reviewed.

\section{REFERENCES}

1 Lisnevskaia L, Murphy G, Isenberg DA. Systemic lupus erythematosus. Lancet 2014:384:1878-88.

2 D'Cruz DP, Khamashta MA, Hughes GR. Systemic lupus erythematosus. Lancet 2007;369:587-96.

3 Murphy G, Lisnevskaia L, Isenberg DA. Systemic lupus erythematosus and other autoimmune rheumatic diseases: challenges to treatment. Lancet 2013;382:809-18.

4 Wallace DJ, Kalunian K, Petri MA, et al. Efficacy and safety of epratuzumab in patients with moderate/severe active systemic lupus erythematosus: results from EMBLEM, a phase $\mathrm{llb}$, randomised, double-blind, placebo-controlled, multicentre study. Ann Rheum Dis 2014;73:183-90.

5 Stohl W. Therapeutic targeting of the BAFF/APRIL axis in systemic lupus erythematosus. Expert Opin Ther Targets 2014;18:473-89.

6 Schneider P, Mackay F, Steiner V, et al. BAFF, a novel ligand of the tumor necrosis factor family, stimulates B cell growth. J Exp Med 1999;189:1747-56.

7 Cancro MP, D'Cruz DP, Khamashta MA. The role of B lymphocyte stimulator (BLyS) in systemic lupus erythematosus. J Clin Invest 2009;119:1066-73.

8 Darce JR, Arendt BK, Wu X, et al. Regulated expression of BAFF-binding receptors during human B cell differentiation. J Immunol 2007;179:7272-86. 
9 Stohl W, Metyas S, Tan S, et al. B lymphocyte stimulator overexpression in patients with systemic lupus erythematosus: Iongitudinal observations. Arthritis Rheum 2003;48:3475-86.

10 Petri M, Stohl W, Chatham W, et al. Association of plasma B lymphocyte stimulator levels and disease activity in systemic lupus erythematosus. Arthritis Rheum 2008:58:2453-9.

11 Hochberg M. Updating the American College of Rheumatology revised criteria for the classification of systemic lupus erythematosus. Arthritis Rheum 1997; 40:1725

12 Petri M, Kim MY, Kalunian KC, et al. Combined oral contraceptives in women with systemic lupus erythematosus. N Engl J Med 2005;353:2550-8.

13 Furie RA, Petri MA, Wallace DJ, et al. Novel evidence-based systemic lupus erythematosus responder index. Arthritis Rheum 2009;61:1143-51.

14 Petri M, Buyon J, Kim M. Classification and definition of major flares in SLE clinical trials. Lupus 1999;8:685-91.

15 Wolfe F. Fatigue assessments in rheumatoid arthritis: comparative performance of visual analog scales and longer fatigue questionnaires in 7760 patients. J Rheumatol 2004;31:1896-902.

16 Mendoza TR, Wang XS, Cleeland CS, et al. The rapid assessment of fatigue severity in cancer patients: use of the Brief Fatigue Inventory. Cancer 1999;85:1186-96.

17 Merrill JT, van Vollenhoven RF, Buyon JP, et al. Efficacy and safety of subcutaneous tabalumab, a monoclonal antibody to B-cell activating factor, in patients with systemic lupus erythematosus: results from ILLUMINATE-2, a 52-week, phase III, multicentre, randomised, double-blind, placebo-controlled study. Ann Rheum Dis 2016;75:332-40

18 Isenberg D, Gordon C, Licu D, et al. Efficacy and safety of atacicept for prevention of flares in patients with moderate-to-severe systemic lupus erythematosus (SLE): 52-week data (APRIL-SLE randomised trial). Ann Rheum Dis 2015;74: 2006-15.

19 Genovese MC, Fleischmann RM, Greenwald M, et al. Tabalumab, an anti-BAFF monoclonal antibody, in patients with active rheumatoid arthritis with an inadequate response to TNF inhibitors. Ann Rheum Dis 2013;72:1461-8.

20 Genovese MC, Lee E, Satterwhite J, et al. A phase 2 dose-ranging study of subcutaneous tabalumab for the treatment of patients with active rheumatoid arthritis and an inadequate response to methotrexate. Ann Rheum Dis 2013;72:1453-60.

21 Greenwald M, Szczepanski L, Kennedy A, et al. A 52-week, open-label study evaluating the safety and efficacy of tabalumab, an anti-B-cell-activating factor monoclonal antibody, for rheumatoid arthritis. Arthritis Res Ther 2014;16:415.

22 Carter LM, Isenberg DA, Ehrenstein MR. Elevated serum BAFF levels are associated with rising anti-double-stranded DNA antibody levels and disease flare following B cell depletion therapy in systemic lupus erythematosus. Arthritis Rheum 2013;65:2672-9.

23 FDA approves Benlysta to treat lupus: First new lupus drug approved in 56 years. FDA. 9 Mar 2011. http://www.fda.gov/newsevents/newsroom/pressAnnouncements/ ucm246489.htm (accessed 13 Feb 2015).

24 Belimumab. Approved indication: systemic lupus erythematosus. Aust Prescr 2013;36:139-42. http://www.australianprescriber.com/magazine/36/4/139/42/ new-drugs/1028/belimumab-for-systemic-lupus-erythematosus (accessed 3 Mar 2015)

25 Navarra SV, Guzmán RM, Gallacher AE, et al. Efficacy and safety of belimumab in patients with active systemic lupus erythematosus: a randomised, placebo-controlled, phase 3 trial. Lancet 2011;377:721-31.

26 Furie R, Petri M, Zamani O, et al. A phase III, randomized, placebo-controlled study of belimumab, a monoclonal antibody that inhibits B lymphocyte stimulator, in patients with systemic lupus erythematosus. Arthritis Rheum 2011;63:3918-30. 